\title{
THE M DWARF ECLIPSING BINARY CU CANCRI
}

\author{
R. E. Wilson ${ }^{1}$, C. A. Pilachowski ${ }^{2,4,5}$, AND Dirk Terrell ${ }^{3}$ \\ ${ }^{1}$ Astronomy Department, University of Florida, Gainesville, FL 32611 and Astronomy Department, Indiana University, \\ Swain Hall West, Bloomington, IN 47405, USA; rewilson@ufl.edu \\ ${ }^{2}$ Astronomy Department, Indiana University Bloomington, Swain West 319, 727 East Third Street, Bloomington, IN 47405-7105, USA; cpilacho@indiana.edu \\ ${ }^{3}$ Department of Space Studies, Southwest Research Institute, 1050 Walnut Street, Suite 300, \\ Boulder, CO 80302, USA; terrell@boulder.swri.edu \\ Received 2016 June 28; revised 2016 October 24; accepted 2016 October 26; published 2017 February 1
}

\begin{abstract}
Spectral features, radial velocities, elemental abundance estimates, other spectral data, and $B V I_{C}$ light curves are reported for the double-M dwarf eclipsing binary CU Cancri-a good target for a radius check versus the Zero Age Main Sequence (ZAMS) due to the low component masses and corresponding very slow evolutionary expansion. The estimate of $[\mathrm{Fe} / \mathrm{H}]$ is about 0.4 , although continuum placement and other difficulties due to line crowding introduce the usual uncertainties for red dwarfs. Detection of the Li I $\lambda 6707$ line was attempted, with an estimated upper limit of $50 \mathrm{~m} \AA$. Spectral and photometric indicators of stellar activity are described and illustrated. Other objectives were to measure the stellar radii via simultaneous velocity and light-curve solutions of earlier and new data while also improving the ephemeris by filling gaps in timewise coverage with the new velocities and eclipse data from the new light curves. The radii from our solutions agree within about $2 \%$ with those from Ribas, being slightly larger than expected for most estimates of the ZAMS. Some aspects of the red dwarf radius anomaly are briefly discussed. Evolution tracks show only very slight age-related expansion for masses near those in CU Cnc. Such expansion could be significant if CU Cnc were similar in age to the Galaxy, but then its Galactic velocity components should be representative of Population II, and they are not.
\end{abstract}

Key words: binaries: eclipsing - binaries: spectroscopic - stars: individual (CU Cancri)

Supporting material: machine-readable table

\section{INTRODUCTION}

Eclipsing binary (EB) $\mathrm{CU}$ Cancri is the " $A$ " component (often designated $\mathrm{Aab}$ ) of a multiple system of at least five $\mathrm{M}$ dwarfs described by Delfosse et al. (1999a) in a search for companions to $127 \mathrm{M}$ dwarfs, many being flare stars, and by Beuzit et al. (2004). See also Haro et al. (1975), Jankovics et al. (1978), and Petersen (1982) for discovery and follow-up on CU Cnc. A common proper motion companion at $12^{\prime \prime}$ separation, the flare star CV Cancri, has been resolved as a binary by adaptive optics (Delfosse et al. 1999a). Its stars are designated the $B$ and $C$ components. Also known by adaptive optics (Delfosse et al. 1999a; Beuzit et al. 2004) is a star designated " $D$ " that is 0 ". 68 from $A$.

The literature on red dwarf stars has been expanding rapidly, partly due to their role as nearly unchanged artifacts of the early universe, partly to challenging observational and structural problems, and partly due to their role as hosts of exoplanets. Flares on red dwarfs are bright relative to the dim luminosities of unevolved low-mass stars, so "flare stars" is an active research area (e.g., see references in Qian et al. 2012 for recent contributions). A practical difficulty for many $\mathrm{M}$ dwarf issues is that their low luminosities strongly limit the distance within which accurate spectroscopy can be done without ultra-large optics. A newly prominent observational area is that of binary M dwarfs-especially ones that eclipse so as to allow accurate measurement of radii and masses. An imaging survey (Janson

\footnotetext{
${ }^{4}$ Visiting astronomer, Apache Point Observatory $3.5 \mathrm{~m}$ telescope, which is owned and operated by the Astrophysical Research Consortium.

${ }^{5}$ Visiting astronomer, Kitt Peak National Observatory, National Optical Astronomy Observatory, which is operated by the Association of Universities for Research in Astronomy (AURA) under a cooperative agreement with the National Science Foundation.
}

et al. 2014) has provided statistics of widely separated (i.e., unlikely to eclipse) red dwarf binaries, while discoveries and light/velocity curve analyses of $\mathrm{M}$ dwarf EBs and EBs with one $\mathrm{M}$ dwarf have been appearing steadily, e.g., Cakirli et al. (2009, 2010, 2013), Dimitrov \& Kjurkchieva (2010), Helminiak et al. (2011), Coughlin et al. (2011), Irwin et al. (2009), Nefs et al. (2013), Gomez et al. (2014), Rozyczka et al. (2009), Zhang et al. (2014), and Zhou et al. (2014). Torres (2013) has reviewed much of this progress with many additional references, as well as comments on observations, analysis, theory, and particular examples.

Only two EBs that consist of a pair of $M$ dwarfs were known prior to publications by Delfosse et al. (1999b) and Ribas (2003) that contained very accurate observations of CU Cnc and called attention to the need for good statistics of lowmass dwarf star properties. The situation has been changing qualitatively and quantitatively over the past decade, perhaps in response to those papers, and also to the flood of light curves from the Kepler and Corot missions (see, e.g., Coughlin et al. 2011). As a result, inroads to several perplexing issues that concern the deep convection zones of red dwarf envelopes can now be more meaningfully addressed. In particular, such binaries are needed to establish the empirical mass-radius relation near zero age for stars with substantial outer convection zones. They thereby allow checks on structural radius computations that require a theory of superadiabatic convection, and thus can guide improvements to that theory. Although only a thin outer region is significantly superadiabatic, that region sets the pressuretemperature relation for the deep underlying zone of adiabatic convection so as to influence the star's radius significantly. Convection-inhibiting phenomena due to small component 
separations and resulting tidally locked fast rotation, such as global magnetic fields (Feiden \& Chaboyer 2013; Macdonald \& Mullan 2014) and local magnetic fields due to heavy spottedness, along with strong irradiation, can influence red dwarf radii. Accordingly an increase in the number of wellobserved and well analyzed examples can drive progress in structural modeling. CU Cnc's components are far enough apart so that irradiation (i.e., the "reflection" effect) is essentially negligible and can be disregarded while considering other effects on star size. Abundance issues-particularly that of lithium - can lead to structural inferences (Feiden \& Chaboyer 2013) and will be touched upon in Section 6, based on high-resolution echelle spectra described and illustrated in Section 2.2. New infrared spectra and radial velocities (RVs) are introduced in Section 2.1 and new $B, V, I_{C}$ light curves are introduced in Section 3. Emission features are described in Section 4 and light/velocity analyses that utilize the Delfosse et al. and Ribas data in simultaneous solutions with the new velocities are in Section 7.

\section{OBSERVATIONS AND DATA REDUCTIONS}

\subsection{Infrared Spectra and Radial Velocities}

Infrared spectra of CU Cnc were obtained with the Phoenix spectrometer on the 2.1 and $4 \mathrm{~m}$ telescopes at Kitt Peak National Observatory in 2013 December, and 2014 January and February. The spectra were centered at $4287 \mathrm{~cm}^{-1}$ $(\lambda=2.33 \mu \mathrm{m})$ using the $4308 \mathrm{~cm}^{-1}$ filter with the $107 \mu \mathrm{m}$ slit, giving a resolving power of $R=50,000$. The spectral region includes numerous (2-0) first overtone lines of $\mathrm{CO}$ and a prominent $\mathrm{Na}$ I feature. The primary star is defined here as the slightly hotter and more massive one that is eclipsed near zero phase according to the ephemeris.

The observations were made using the "abba" mode with two slit positions to facilitate synchronous sky subtraction. Data reductions followed standard procedures with IRAF. ${ }^{6}$ Images were subtracted in pairs, and the one-dimensional spectra were extracted. This observing mode yields four independent observations per night spanning approximately one hour. Early-type stars were observed to permit removal of the telluric line spectrum with the IRAF task "telluric." The telluric spectrum provided an accurate wavelength calibration.

The RVs of the two components were determined with the IRAF task "fxcor" by cross-correlating each wavelengthcalibrated spectrum against a sunspot umbra spectrum from the Wallace \& Livingston (1992) atlas. The umbral spectrum was convolved with a Gaussian and re-sampled to match the resolution of our Phoenix data. The umbral spectrum, with a temperature near $4200 \mathrm{~K}$, is dominated by the same $\mathrm{CO}$ features that dominate the $\mathrm{CU} \mathrm{Cnc}$ spectrum in this region. Near 2.3 microns, the spectrum is much simpler than in the optical, with many fewer features. As shown in Figure 1 and despite the difference in temperature, the umbral spectrum is a remarkably close match to the $\mathrm{CU}$ Cnc spectrum. In this context, the infrared differs considerably from the optical region, where such a large temperature difference usually results in very different spectra. The similarity of the spectra might result from the gravity sensitivity of the CO lines,

\footnotetext{
6 The Image Reduction and Analysis Facility (IRAF) is software distributed by the National Optical Astronomy Observatory, which is operated by the Association of Universities for Research in Astronomy, Inc., under cooperative agreement with the National Science Foundation.
}

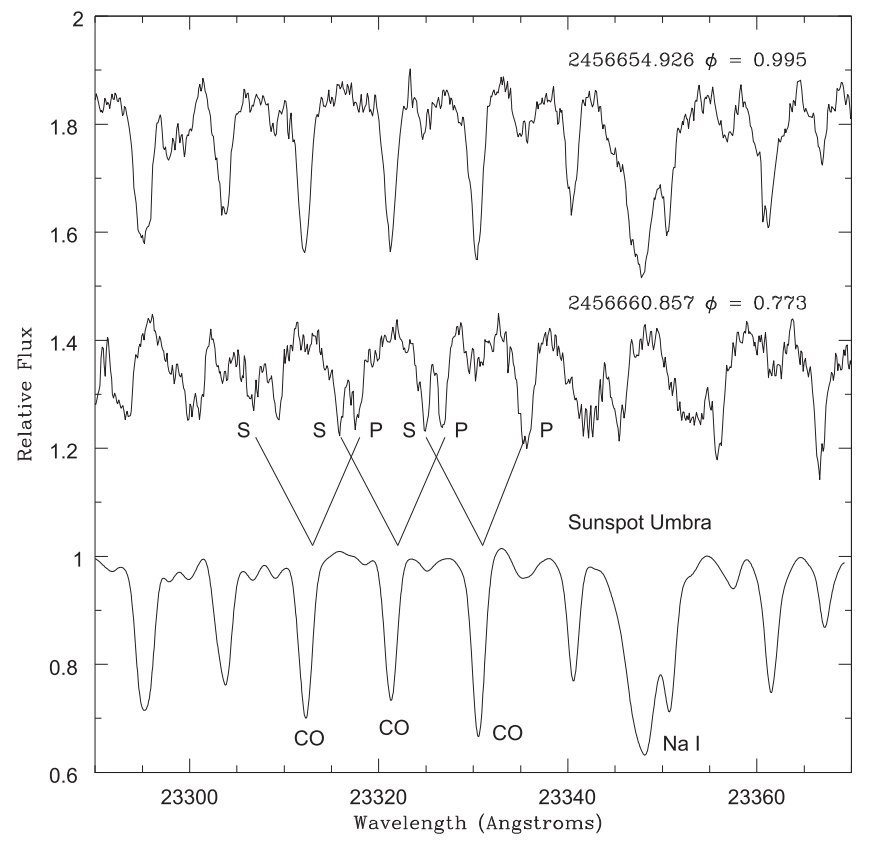

Figure 1. Infrared spectra of $\mathrm{CU} C \mathrm{Cnc}$ at two phases, together with the spectrum of a sunspot umbra from the Wallace \& Livingston (1992) atlas at the bottom. The umbral spectrum has been convolved with a Gaussian to produce a resolution similar to that of our CU Cnc spectra. The upper spectrum has been shifted upward by 0.85 in relative flux, while the middle spectrum has been shifted upward by 0.4 . The upper spectrum, at phase 0.995 closely resembles the umbral spectrum, while the spectral lines of the two components are clearly resolved in the middle spectrum, obtained at phase 0.772 . Features due to the primary and secondary stars are indicated by the symbols $\mathrm{P}$ and $\mathrm{S}$. The strong, regularly spaced doublet features in the umbral spectrum are due to lines of the (2-0) overtone of the molecule CO. The strong, broad feature at $23348 \AA$ is due to Na I.

whereby the lower gravity in a sunspot compared to an M4 dwarf might compensate for the temperature effect. In any case, the close match between the umbral and $\mathrm{CU}$ Cnc spectra supports the use of the umbral spectrum as an appropriate template for the much cooler $\mathrm{M}$ dwarfs.

Given the relatively high signal-to-noise ratio $(\mathrm{S} / \mathrm{N} ; \mathrm{S} / \mathrm{Ns}$ exceed 50/1 and for some spectra, exceed $100 / 1)$ and resolving power of our spectra $(R=50,000)$, the crosscorrelation peaks are sharp, well-defined, and well-fit by Gaussians. At phases where the velocity difference between the two components is small, the cross-correlation peak was deblended by fitting two Gaussians. Near conjunction, where the features could not be deblended, velocities are not reported. Figure 1 includes two sample spectra from our $2.1 \mathrm{~m}$ telescope observations along with the umbral template spectrum. They show phases with both a large and a small RV difference between components.

The solutions of Section 7 are simultaneous in light and RV and deliver one systemic velocity, $V_{\gamma}$, not a $V_{\gamma}$ for each $\mathrm{CU}$ Cnc component. A side exercise to carry out separate star 1 and 2 solutions of our RV's found $V_{\gamma}$ 's that differ by $1.59 \pm 0.26 \mathrm{~km} \mathrm{~s}^{-1}$, so they are unequal at about the $6 \sigma$ level. Such small but formally improbable differences in $V_{\gamma}$ 's that should be essentially the same can arise from measurement difficulties in binary spectra with phase-variable blending. The Delfosse et al. (1999b) data, when treated the same way, give a $V_{\gamma}$ difference for the components of only $0.25 \pm 0.17 \mathrm{~km} \mathrm{~s}^{-1}$, well within $1 \sigma$. The older and newer RV's, entered as one data 
Table 1

Radial Velocity Observations with the Phoenix Spectrometer

\begin{tabular}{|c|c|c|c|c|c|}
\hline HJD-2450000 & $\begin{array}{l}\text { Exp. Time } \\
\text { (s) }\end{array}$ & $\begin{array}{l}\text { RV Primary } \\
\left(\mathrm{km} \mathrm{s}^{-1}\right)\end{array}$ & $\begin{array}{c}\text { RV Secondary } \\
\left(\mathrm{km} \mathrm{s}^{-1}\right)\end{array}$ & Tonry-Davis " $R$ " & $\begin{array}{c}\sigma \\
\left(\mathrm{km} \mathrm{s}^{-1}\right)\end{array}$ \\
\hline 56656.88971 & 900 & 67.31 & -65.52 & 7.4 & 1.1 \\
\hline 56656.90043 & 900 & 67.15 & -67.82 & 8.8 & $\ldots$ \\
\hline 56656.92177 & 900 & 69.55 & -66.81 & 8.2 & $\ldots$ \\
\hline 56658.87022 & 900 & -29.33 & 40.18 & 8.6 & 1.4 \\
\hline 56658.88094 & 900 & -28.69 & 38.73 & 8.4 & $\ldots$ \\
\hline 56659.83516 & 900 & 71.96 & -67.73 & 8.3 & 0.7 \\
\hline 56659.84587 & 900 & 70.42 & -67.70 & 4.2 & $\ldots$ \\
\hline 56659.85649 & 900 & 70.45 & -67.20 & 7.1 & $\ldots$ \\
\hline 56659.86722 & 900 & 71.04 & -66.14 & 8.2 & $\ldots$ \\
\hline 56659.88594 & 900 & 69.70 & -66.38 & 7.3 & 1.0 \\
\hline 56659.89665 & 900 & 68.99 & -64.93 & 4.5 & $\ldots$ \\
\hline 56659.95048 & 900 & 67.11 & -64.24 & 19 & $\ldots$ \\
\hline 56659.96084 & 900 & 66.15 & -63.99 & 17 & $\ldots$ \\
\hline 56660.82573 & 900 & -44.90 & 57.02 & 6.9 & 1.4 \\
\hline 56660.83645 & 900 & -47.03 & 57.85 & 7.0 & $\ldots$ \\
\hline 56660.84707 & 900 & -48.23 & 58.67 & 7.9 & $\ldots$ \\
\hline 56660.85779 & 900 & -46.91 & 61.29 & 10 & $\ldots$ \\
\hline 56660.86871 & 900 & -49.34 & 62.40 & 11 & 0.9 \\
\hline 56660.87942 & 900 & -51.03 & 62.23 & 7.7 & $\ldots$ \\
\hline 56660.89005 & 900 & -51.39 & 63.26 & 14 & $\ldots$ \\
\hline 56660.90076 & 900 & -50.28 & 65.11 & 9.9 & $\ldots$ \\
\hline 56660.91167 & 900 & -52.28 & 65.59 & 11 & 1.4 \\
\hline 56660.92238 & 900 & -54.93 & 64.41 & 8.3 & $\ldots$ \\
\hline 56660.93300 & 900 & -54.95 & 64.49 & 11 & $\ldots$ \\
\hline 56705.85360 & 600 & -48.21 & 59.00 & 9.0 & $\ldots$ \\
\hline 56706.83210 & 600 & 68.87 & -68.36 & 7.1 & 0.6 \\
\hline 56706.83944 & 600 & 70.27 & -68.56 & 9.1 & $\ldots$ \\
\hline 56706.84675 & 600 & 69.78 & -69.42 & 6.9 & $\ldots$ \\
\hline 56706.85410 & 600 & 69.37 & -70.43 & 7.1 & $\ldots$ \\
\hline 56708.83602 & 600 & -19.05 & 27.78 & 8.1 & 1.1 \\
\hline 56708.84338 & 600 & -18.57 & 26.67 & 7.0 & $\ldots$ \\
\hline 56708.85068 & 600 & -17.02 & 25.42 & 7.2 & $\ldots$ \\
\hline 56708.85803 & 600 & -17.01 & 23.38 & 7.2 & $\ldots$ \\
\hline
\end{tabular}

Note. Eight additional spectra from very near the conjunctions were made. The corresponding RV's are not reported due to obvious or likely line blending.

set with proper weighting for each component, give a $V_{\gamma}$ difference of $0.11 \pm 0.16 \mathrm{~km} \mathrm{~s}^{-1}$, where the standard error is a root-mean-square sum of the separate errors.

The RVs, midpoint heliocentric Julian Dates, and integration times are in Table 1, along with the Tonry \& Davis (1979) " $R$ " value for each observation and the standard deviations of the four independent measurements for each night. The $R$ value is the height of the cross-correlation peak divided by the noise in the cross-correlation function. Observations with $R$ 's above three are ordinarily considered reliable.

\subsection{Echelle Spectra}

High-resolution optical spectra of CU Cnc were obtained for estimation of elemental abundances (see Section 6). An $1800 \mathrm{~s}$ observation was obtained on 2014 May 17 with the Astrophysical Research Consortium (ARC) echelle spectrograph (ARCES) at a phase near quadrature. A second spectrum was obtained with the same instrument on 2015 November 21 with an exposure time of $3600 \mathrm{~s}$ at a phase near conjunction. The spectra cover the wavelength range from 3500 to $10,000 \AA$ and resolving power is about 31,500 ( 2.5 pixels) with the $1 . " 6 \times 3$ ". 2 slit. The midpoint 
Table 2

Emission Feature Equivalent Widths

\begin{tabular}{lcc}
\hline \hline Feature & $\begin{array}{c}\text { Primary } \\
(\AA)\end{array}$ & $\begin{array}{c}\text { Secondary } \\
(\AA)\end{array}$ \\
\hline $\mathrm{H} \alpha$ & -2.58 & -2.61 \\
$\mathrm{H} \beta$ & -3.29 & -3.51 \\
$\mathrm{H} \gamma$ & -2.75 & -2.95 \\
$\mathrm{H} \delta$ & -1.51 & -1.69 \\
$\mathrm{He}$ I $\lambda 5877$ & -0.27 & -0.31 \\
$\mathrm{Na} \mathrm{I} \lambda 5890$ & -0.59 & -0.43 \\
$\mathrm{Na} \mathrm{I} \lambda 5896$ & -0.38 & -0.29 \\
\hline
\end{tabular}

HJD of the 2014 exposure was 2456794.6458 , corresponding to a phase of 0.41 , when the components' velocities are well separated; The phase at midpoint of the 2015 exposure is 0.04 , when the velocity separation of the stars is small. The spectra were bias corrected, flat-fielded, cleaned, and extracted to wavelength-calibrated one-dimensional spectra with IRAF. At wavelengths longer than $5000 \AA$ the $\mathrm{S} / \mathrm{N}$ of the final reduced 2014 spectrum is typically 75 near the center of the orders, but declines to near 50 at the edges and at shorter wavelengths; the $\mathrm{S} / \mathrm{N}$ of the 2015 spectrum is 100 near the centers of the echelle orders.

\section{LIGHT CURVES IN $B, V$, AND $I_{C}$ BANDS}

The CU Cnc field was observed at the Sonoita Research Observatory with a $0.5 \mathrm{~m} f / 4$ Newtonian telescope and a Santa Barbara Instrument Group STXL-6303 CCD camera, with Johnson-Cousins $B V I_{C}$ and SDSS $g^{\prime} r^{\prime} i^{\prime}$ filters. The images were first processed in the usual way by subtracting bias and dark frames, and then flatfielding in IRAF. Purposes were to supplement the Ribas (2003) data with CU Cnc light curves at another epoch and to measure Johnson $R, I$ magnitudes for the Ribas comparison star, HD 72093, to allow for the conversion of his differential measures to standard magnitudes.

CU Cnc aperture photometry was done in IRAF, with the $B V I_{C}$ differential magnitudes against GSC 1387-01006 extending from JD 2457006 to 2457081. The light curves are in Table 3. They were used here only to fine-tune the ephemeris (see Section 7) since they show small flares, especially in $B$ and $V$, but they may be of interest to others for statistics of flaring. The aperture radius at the CCD was 7 !. 35, thus excluding the BC component, CV Cnc. A more complicated process was necessary for calibration of Ribas' comparison star, HD 72093, in $R$ and $I$. Johnson $R I$ filters were not available so the field was observed in Johnson $B V$ and SDSS $g^{\prime} r^{\prime} i^{\prime}$. The instrumental magnitudes were then transformed to the standard systems via on-chip standards from Data Release 9 of the AAVSO Photometric All-Sky Survey (Henden et al. 2012), observed simultaneously with HD 72093. The data then entered a threestep process to compute the Johnson RI magnitudes. First, the SDSS website gives transformations from the $g^{\prime} r^{\prime} i^{\prime}$ system to the gri system. Then Jester et al. (2005) give transformations between Cousins and SDSS gri colors, thus allowing the formation of $R_{C}-I_{C}$ and $V-I_{C}$. Finally, Bessell (1979) gives transformations between Johnson $R I$ and Cousins $R_{C} I_{C}$ that can put the Ribas (2003) differential observations onto standard scales. $R$ and $I$ for HD 72093 were measured to be $7^{\mathrm{m}} \cdot 340 \pm 0^{\mathrm{m}} \cdot 024$ and $6^{\mathrm{m}} \cdot 962 \pm 0^{\mathrm{m}} \cdot 018$, respectively.

\section{STELLAR ACTIVITY}

Our 2014 and 2015 ARCES spectra show strong emission features from both components of $\mathrm{CU} \mathrm{Cnc}$ for $\mathrm{H} \alpha$ through $\mathrm{H} \delta$, as well as emission at He I $\lambda 5877 \AA$ and the $\mathrm{Na} D$ lines at $\lambda 5890$ and $\lambda 5896 \AA$. Equivalent widths are in Table 2 . The emission features of the two stars in our 2014 spectrum, taken at phase 0.41 , are separated by $77 \mathrm{~km} \mathrm{~s}^{-1}$. Each component's $\mathrm{H} \alpha$ equivalent width is $2.6 \AA$, somewhat less than Ribas found from his 2001 observation. Both $\mathrm{H} \alpha$ emission features are clearly double peaked, with separations of $0.67 \AA$ for the primary and $0.69 \AA$ for the secondary, corresponding to a velocity difference of $31 \mathrm{~km} \mathrm{~s}^{-1}$. The $\mathrm{H} \beta, \mathrm{H} \gamma, \mathrm{H} \delta, \mathrm{He}$, and $\mathrm{Na}$ $\mathrm{D}$ profiles do not appear double peaked. The $\mathrm{H} \alpha$ profiles are shown in Figure 2.

Double-peaked $\mathrm{H} \alpha$ emission is common in slowly rotating M dwarfs. Stauffer et al. (1997) provide examples of four Hyades M dwarfs with $V \sin i$ less than $7 \mathrm{~km} \mathrm{~s}^{-1}$ that show similar double-peaked emission profiles, and cite an explanation by Cram \& Mullan (1985) that the profiles are due to nonthermal chromospheric velocity fields.

\section{SPECTROSCOPIC RATIOS OF LUMINOSITIES AND RADII}

As emphasized by Ribas (2003), a definite ratio of the component bandpass luminosities from information external to the light curves can improve solutions for CU Cnc-like EB's. The reason is that light-curve solutions do not give reliable individual radii, $R_{1,2} / a$, for such partially eclipsing configurations but only their sum, which basically comes from eclipse duration as a fraction of the period. The situation would be better for total-annular eclipses, where durations of the complete eclipse intervals can essentially measure the difference of $R_{1} / a$ and $R_{2} / a$, while in some total-annular examples the eclipse depths can measure $R_{2} / R_{1}$, but CU Cnc's eclipses are partial (and shallow). Of course, the average from a properly computed pair of radii can be adopted as the average of the true radii when the sum of the radii is accurately measured-thus not leading statistically to over- or underestimates of average radius.

A spectroscopic luminosity ratio that pertains to a spectral region can be estimated from the ratio of line equivalent widths since CU Cnc's star temperatures are nearly equal. Ribas measured $L_{2} / L_{1}$ at wavelength $0.59 \mu \mathrm{m}$ and then ran a sequence of solutions for stepped primary star surface potential. He thereby established a sequence of surface potential pairs, along with other parameters, and settled on the solution that agreed with the spectroscopic luminosity ratio. That solution, with its definite surface potentials, then has definite star radii. Our procedure was similar and our solutions adopted Ribas' estimates for $L_{2} / L_{1}$ in the $R, I$ bands.

For possible future use, we also estimated $L_{2} / L_{1}$ from line equivalent widths from our infrared spectra at $\approx 2.33 \mu \mathrm{m}$ wavelength. From five spectra with well separated component lines, 17 pairs of equivalent widths yield $L_{2} / L_{1}=0.90 \pm 0.03$ (standard error of the mean). In addition to the infrared measurement, we also selected 13 clean, unblended lines in the 2014 echelle spectrum from the ARC $3.5 \mathrm{~m}$ telescope in the wavelength region from 0.78 to $0.87 \mu \mathrm{m}$ in several orders. The line blanketing in this region is reduced compared to other parts of the spectrum, and the continuum was set at the lowest height consistent with nearby, line-free regions. The average of the 13 
Table 3

Light Curves in the $B, V$, and $I_{C}$ Bands

\begin{tabular}{lcccr}
\hline \hline HJD & $\delta B$ & HJD & $\delta V$ & HJD \\
\hline 2456986.7883 & 0.112 & 2456986.7869 & -0.661 & 2456986.7856 \\
2456986.7896 & 0.148 & 2456986.7909 & -0.661 & 2456986.7921 \\
2456986.7959 & 0.101 & 2456986.7946 & -0.687 & 2456986.7934 \\
2456986.7973 & 0.147 & 2456986.7986 & -0.669 & 2456986.7998 \\
\hline
\end{tabular}

(This table is available in its entirety in machine-readable form.)

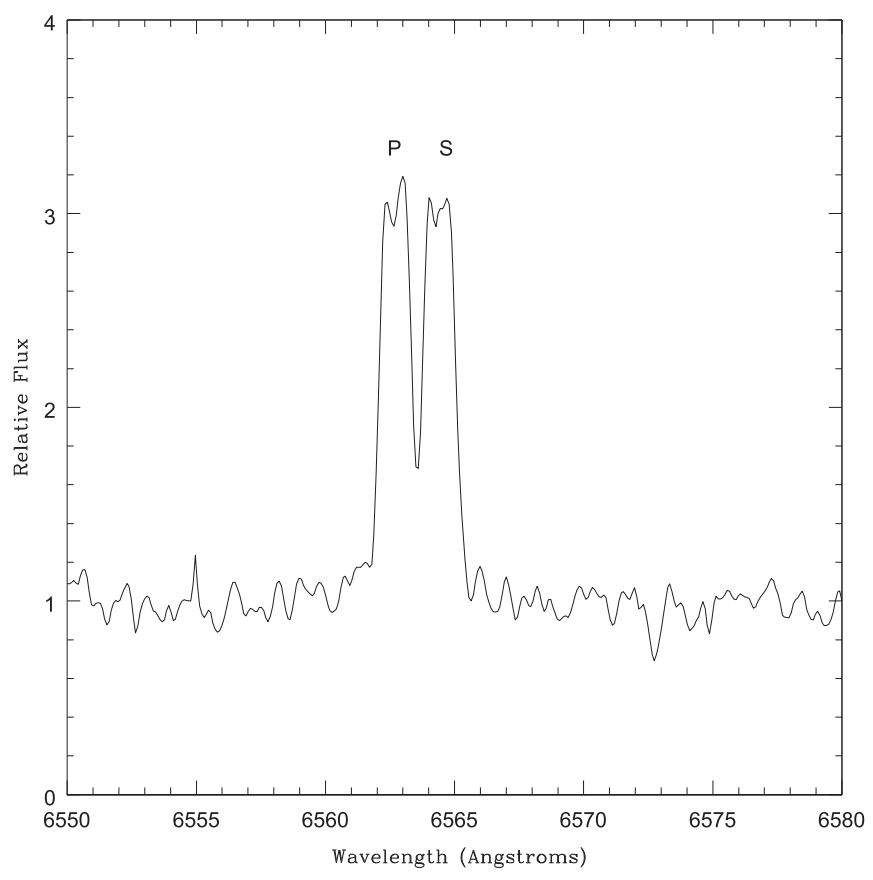

Figure 2. ARC echelle spectrum of the $\mathrm{H} \alpha$ feature in $\mathrm{CU}$ Cnc at phase $=0.41$. The emission feature from the primary star is shifted toward shorter wavelengths at this phase. Emission from both the primary and the secondary stars is double peaked.

lines is $L_{2} / L_{1}=0.84 \pm 0.03$ (standard error of the mean). This result compares to measurements by Ribas of $L_{2} / L_{1}=0.72$, 0.74 , and $0.76 \pm 0.05$ in the $V, R$, and $I$ bands, respectively. Our measurements are plotted along with the Ribas luminosity ratios versus wavelength in Figure 3. Placement of the continuum is increasingly difficult at shorter wavelengths, and the systematic error introduced by too-low continuum placement likely increases, resulting in lower values for $L_{2} / L_{1}$ based on equivalent width ratio. Given the relatively high $\mathrm{S} / \mathrm{N}$ of the IR spectra and the availability of continuum, we suggest that $L_{2} / L_{1}$ may be higher than previously estimated. A solution at $\approx 2.33 \mu \mathrm{m}$ cannot be carried out without a light curve at that wavelength, although the extended trend of luminosity ratio with wavelength shows that star 2 is definitely cooler than star 1.

\section{ABUNDANCES}

The derivation of absolute abundances in $\mathrm{M}$ dwarfs remains challenging, and we only attempt a crude estimate here. The spectrum analysis code of Sneden (1973) was used with MARCS model atmospheres adopted from the grid of Gustafsson et al. (2008).

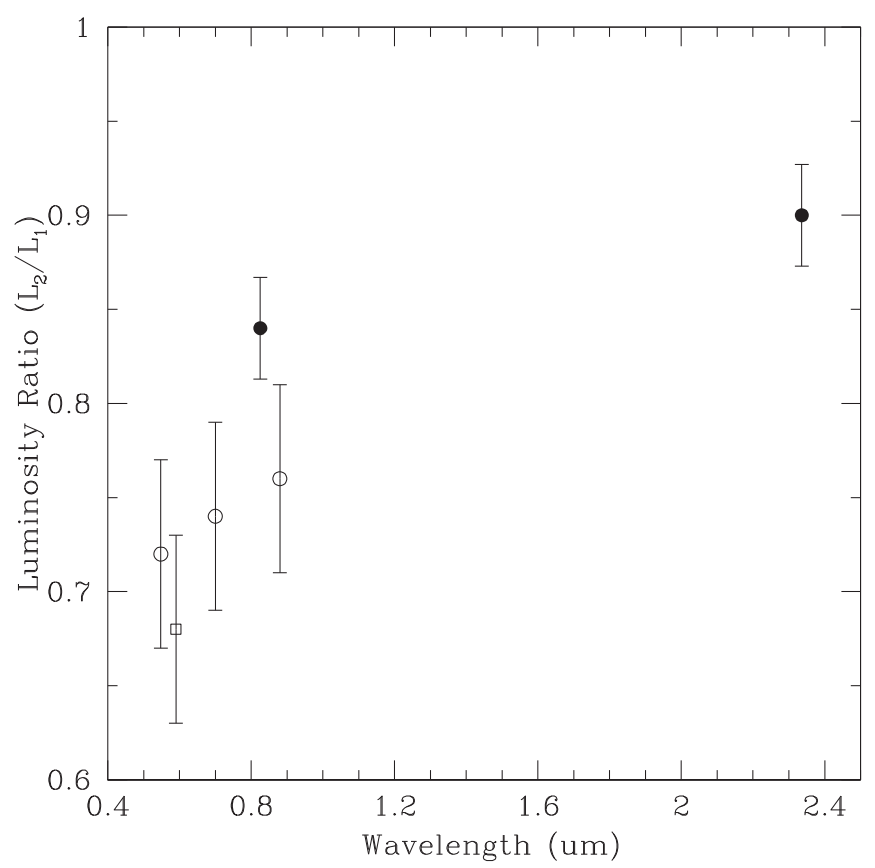

Figure 3. Spectroscopic and spectroscopic-photometric luminosity ratio $\left(L_{2} /\right.$ $L_{1}$ ) vs. wavelength. Open circles: sp./ptm. by Ribas (2003); open square: sp. by Ribas (2003) from Delfosse et al. (1999b) data; filled circles: sp. for this paper.

\subsection{Metallicity}

Ribas (2003) examined CU Cnc's metallicity from isochrone and kinematic viewpoints, with reference to an earlier kinematic estimate (Delfosse et al. 1999b) of $[M / H]=+0.5$, and argued for $[\mathrm{Fe} / \mathrm{H}]$ being about 0.0 . Metallicity measures from line equivalent widths are very difficult for $M$ stars due to uncertainties in continuum levels. Thus we selected a reasonably isolated iron line at $8611.8 \AA$ in a region that enjoys somewhat reduced line densities compared to other parts of the spectrum. The effective local continuum in CU Cnc was set at the lower limit of what might be real-of course a higher setting would increase the derived iron abundance. The measured line equivalent width is $133 \mathrm{~m} \AA$. The Vienna Atomic Line Database (Ryabchikova et al. 2015) provides an excitation potential of $2.84 \mathrm{eV}$ and $\log g f=-1.92$. This value was adjusted to $\log g f=-2.10$ through comparison with the solar spectrum (Wallace et al. 1993). The computation was done for two temperatures, 3100 and $3200 \mathrm{~K}$, with similar results suggesting $[\mathrm{Fe} / \mathrm{H}] \approx 0.4$. Accordingly, $[M / H]=0.5$ was adopted for all of our light/velocity solutions. A pair of light/velocity solutions with $[M / H]=0.0$ and 0.5 and otherwise identical input gave only very slightly different 

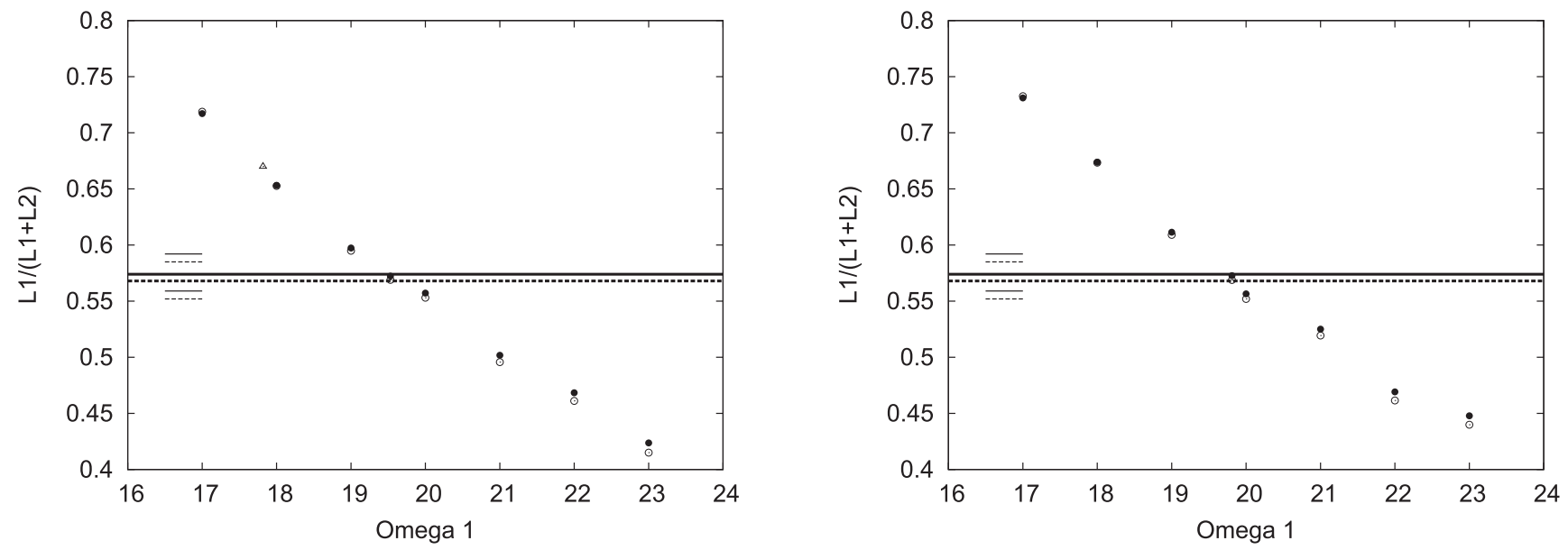

Figure 4. CU Cnc luminosity ratios, $L_{1} /\left(L_{1}+L_{2}\right)$, in Johnson bands $R$ (dots) and $I$ (circles) from the stepped simultaneous light-velocity solutions of Section 7. Light from the multiple system's $D$ component is not modeled in the left panel and properly treated in the right panel. Horizontal lines are placed at the fractional luminosities found by Ribas (2003) from Ca I and Fe I equivalent widths (unbroken for $R$ band, dashed for $I$ band). The thinner lines near the left side indicate the $1 \sigma$ ranges estimated by Ribas for $L_{1} /\left(L_{1}+L_{2}\right)$. The intersection of the diagonal and horizontal relations marks where the computed and observed luminosity ratios agree. Although the diagonal relations are shifted relatively, the crossing points lead to the same solution results for most parameters. The Case I and Case II radii $\left(R_{1,2} / R_{\odot}\right)$ differ by a few $\sigma$, but not enough to see easily in Figure 7. The triangle in the left panel marks the best light/velocity curve fit.

results, so metallicity is not an important issue for CU Cnc's light/velocity solutions.

\subsection{Lithium}

Our high-resolution spectrum was examined for the Li I $\lambda 6707$ line. At the observed phase, the wavelength difference of the lithium features of the two components should be $1.7 \AA$, corresponding to a velocity difference of about $77 \mathrm{~km} \mathrm{~s}^{-1}$, with the primary shifted to a shorter wavelength. The $6707 \AA$ region is heavily obscured by numerous $\mathrm{TiO}$ features and the continuum is not discernable. Synthesis of the Li I feature in a single $\mathrm{M}$ dwarf with a temperature of $3100 \mathrm{~K}$ suggests that a lithium abundance of $\log \epsilon(\mathrm{Li})=1.0$ should produce an equivalent width of nearly $500 \mathrm{~m} \AA$. The echelle spectrum from 2015 , taken at phase 0.04 when the relative velocity shift between the two binary components is small, shows no measurable feature at the location of the Li I doublet. Ribas (2003) suggests a detection of the Li I feature in both components with an equivalent width of $50 \mathrm{~m} \AA$, but we are unable to confirm that detection from our spectra with any confidence. We concur with an upper limit of $50 \mathrm{~m} \AA$ for the Li I feature and with the conclusion of Ribas (2003) that lithium is substantially, if not fully, depleted.

\section{SIMULTANEOUS LIGHT AND VELOCITY CURVE ANALYSES}

\subsection{Initial Reconnisance}

Our analyses utilized the W-D binary system computer model (Wilson \& Devinney 1971; Wilson 1979, 1990, 2007, 2008; Wilson \& Van Hamme 2009, 2014; Wilson et al. 2010; Wilson 2012a) and its Differential Corrections (hereafter DC) program. One might expect $\mathrm{CU} \mathrm{Cnc}$ to be an easy example for light-curve solutions, judging from its well-detached morphology, circular orbit, and lack of major complications. The limb should be sharp, as extended atmospheres are not expected and the stars are almost spherical. However, as stressed by Ribas (2003; see Section 5), the solution is not so easy. Although the Ribas light curves are rather precise (note the stretched scale and small brightness range), essentially no geometric information comes from outside the narrow, partial, and shallow eclipses. There is modest spot activity but that makes analysis slightly weaker, not stronger. ${ }^{7}$ As with many binaries that have shallow partial eclipses, CU Cnc's overall eclipse durations basically tell the sum of the star radii but the eclipses give no other relation between the radii, such as their difference or ratio. Ribas (2003) recognized these difficulties and minimized their effects by running a sequence of trial solutions for stepped star 1 radii, and we did the same. Indeed, all of our stepped solutions (Section 7.7) give $R_{1}+R_{2}$ near $0.800 R_{\odot}$ over a substantial range in $R_{1}$, while derived individual radii are sensitive to minor changes in procedures and parameter values.

\subsection{Ephemeris}

The solutions of Table 4 may have improved the ephemeris by including the RV curves from JD 2,456,656 to 2,456,708 in Table 1 that are well separated in time from the Delfosse et al. (1999b) velocities (JD 2,450,102 to 2,450,923) and Ribas (2003) light curves (JD 2,451,152 to 2,451,262). Our derived period, with input of the new RVs but not the new light curves, is $2.7714700 \pm 0.0000021$, compared to Delfosse et al.'s $2.771468 \pm 0.000004$, so the new RVs do somewhat reduce the formal uncertainty. Our dimensionless $d P / d t$ is $-6.7 \pm 5.9 \times 10^{-10}$. This (at best) marginal $d P / d t$ result is a first attempt for $\mathrm{CU} \mathrm{Cnc}$, made possible in the absence of published eclipse timings by unified light and velocity solutions (Wilson 1979; see also Section 5 of Wilson 2005 and Section 4 of Wilson \& Van Hamme 2014). The $B V I_{C}$ light curves of Section 3 cover 75 days, beginning about 300 days after completion of the next latest curves, thus extending the timewise baseline. Although the $B V I_{C}$ curves are affected by flare activity (Qian et al. 2012), the needle-like eclipses remain good timing ticks, so we checked whether they made a

\footnotetext{
Windmiller et al. (2010) comment in their introduction that W-D's spot facility "...is somewhat simplistic," supposedly with only one or two circular regions. Actually, W-D accomodates any number of adjustable spots, while Windmiller et al.'s (2010) spot parameters are the same as W-D's and Windmiller et al. apply only a few spots, so their spot model is not more realistic than W-D's.
} 

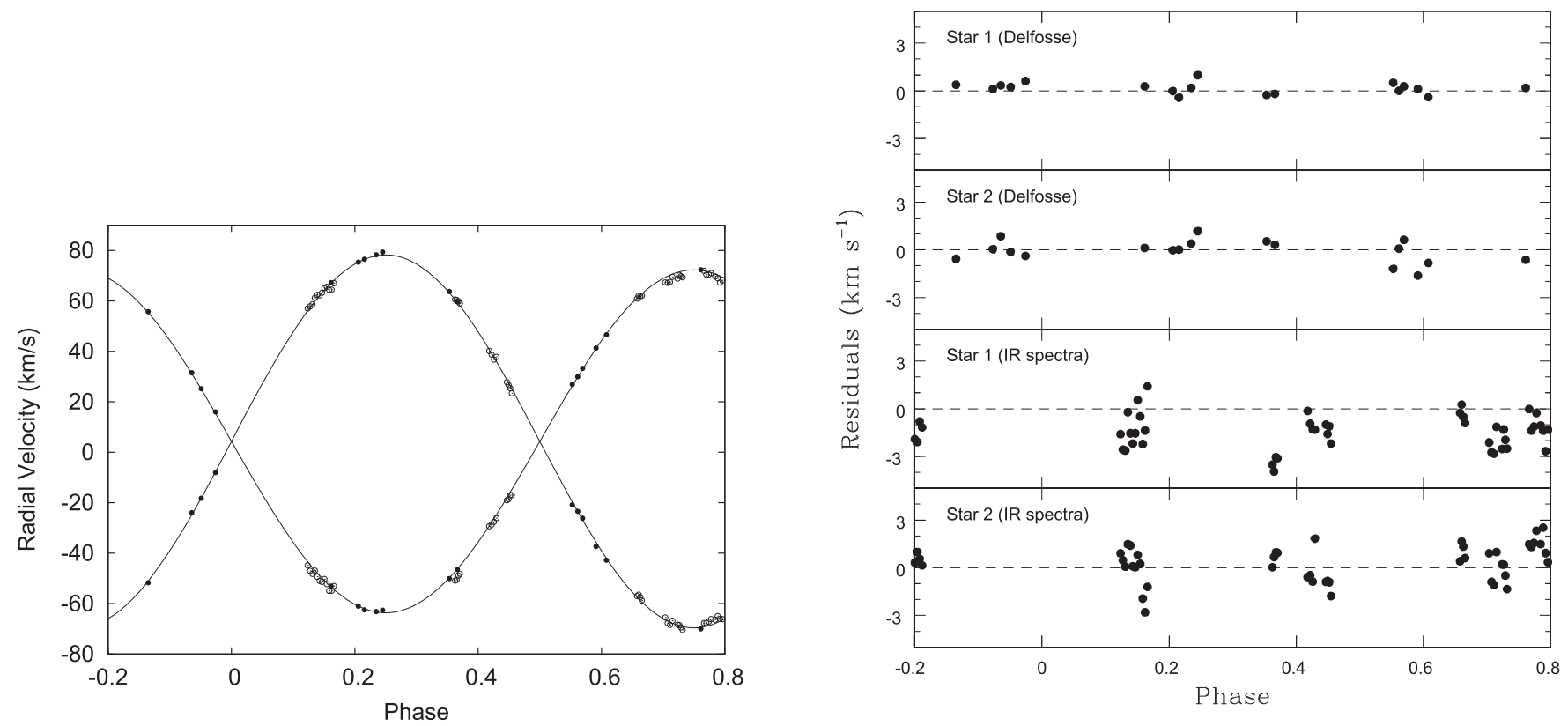

Figure 5. Left panel: CU Cnc radial velocities (dots) by Delfosse et al. (1999b) and those of our Table 1 (circles), along with computed curves based on Case II of Table 4. Right panels: velocity residuals on a stretched scale (upper and lower borders are at +5 and $-5 \mathrm{~km} \mathrm{~s}^{-1}$ in the vertically arranged panels).

noticeable change in the derived ephemeris. With only the $B V I_{C}$ eclipse data and a few points immediately outside eclipse added to the previous solution input (i.e., disturbances by flares reduced as much as practicable), the reference epoch, orbit period, and their standard errors were virtually as in Table 4 . However, $d P / d t$ did change slightly to $-6.1 \pm 5.1 \times 10^{-10}$. Perhaps worth noting is that every one of our solutions, whether explorative or adopted (tabulated), gave a negative $d P / d t$ of about this size that differed from zero by a little more than $1 \sigma$. Future CCD or photomultiplier eclipse timings, RV curves, or light curves could be processed along with those entered here to tighten the uncertainty in $d P / d t$ via the ideas in Wilson \& Van Hamme (2014).

\subsection{Spotted Regions}

The light curves show spot effects, as expected for lowtemperature stars. The solution process was broken into two parts (first the spot parameters, then all other parameters) to avoid having an excessive number of parameters under adjustment together. Solutions began with subjective spot trials that produced approximate agreement in $R$ and $I$, followed by DC solutions for final longitudes, radii, and temperatures of two spots, both on star 1. Spot latitudes were set to values deemed reasonable. No indication of spot drift or growth and decay was evident when the light curves were broken into four time intervals. The resulting spot parameters were kept fixed in the subsequent DC solutions for other parameters, and are in Table 4. Agreement of the observed and computed spot-related variations seems good in both Johnson bands except for some departure in the maximum following secondary eclipse in the $R$ band. That shortcoming could probably be overcome by the introduction of a third spot (or spot region), but is unlikely to compromise results on star dimensions and other properties.

Numerical experiments by Morales et al. (2010) that are too extensive to summarize here examined spot effects on derived EB parameters, including star radii. If spots are not modeled, Morales et al. find typical radius changes of about 3\% for some distributions, although those results are for stronger spottedness than in Ribas' CU Cnc light curves. Morales et al. have many figures of light curves that are affected by spots and also figures of light-curve amplitude versus a spot distribution parameter. They did not systematically address radius differences among various "spotted" solutions-an extension that would have required many more figures and probably also more tables. Of course, solutions that do introduce modeled spots, even if less than ideally, should give radius changes much smaller than the $3 \%$ corresponding to the complete neglect of spots. All of our (and Ribas') solutions included spots and evaluated their parameters by the least squares criterion. Windmiller et al. (2010) investigated star radius dependence on spottedness for GU Boo, a more massive EB than CU Cnc with much deeper eclipses, and found $R_{1}+R_{2}$ nearly the same between a heavily spotted and a lightly spotted epoch.

\subsection{Timewise Spot Changes?}

The $R, I$ light-curve observation times fall between those of the two RV data sets with no overlap. Although light-curve plots at approximately 28-day time steps give no clear evidence of spot growth and/or decay, there could have been changes over the much longer intervals between the light and $\mathrm{RV}$ curves. A direct check of this possibility by examination of the RV's is not feasible since spots influence RV's only very subtly, but we feel that spots of the light-curve era should not be forced upon the RV's. Accordingly, the spots were "turned on" about 50 days prior to the start of the light-curve data (in an observational gap) and "turned off" a similar length of time after the end of the light-curve data. This treatment is a special case of the spot growth and decay algorithm that is given quantitatively in Section 3 of Wilson (2012b). The modeled starspots, therfore, were constant over the light-curve data and there were no spots during the two $\mathrm{RV}$ time ranges. 
Table 4

CU Cancri Simultaneous Light/Velocity Curve Solutions

\begin{tabular}{|c|c|c|c|}
\hline Parameter & Case I & Case II & Ordinary Soln. \\
\hline$a\left(R_{\odot}\right)$ & $7.7863 \pm 0.0089$ & $7.7851 \pm 0.0089$ & $7.7858 \pm 0.0089$ \\
\hline$V_{\gamma}\left(\mathrm{km} \mathrm{s}^{-1}\right)$ & $4.335 \pm 0.059$ & $4.334 \pm 0.059$ & $4.335 \pm 0.059$ \\
\hline$i(\mathrm{deg})$ & $86.414 \pm 0.010$ & $86.537 \pm 0.010$ & $86.489 \pm 0.012$ \\
\hline$T_{2}(\mathrm{~K})$ & $3085 \pm 2$ & $3080 \pm 0.0002$ & $3081 \pm 2$ \\
\hline$\Omega_{1}$ & 19.530 & 19.810 & $17.818 \pm 0.063$ \\
\hline$\Omega_{2}$ & $19.630 \pm 0.086$ & $19.835 \pm 0.088$ & $22.06 \pm 0.12$ \\
\hline$P_{0}(\mathrm{~d})$ & $2.7714701 \pm 0.0000021$ & $2.7714700 \pm 0.0000021$ & $2.7714702 \pm 0.0000021$ \\
\hline$d P / d t$ & $-6.8 \pm 5.9 \times 10^{-10}$ & $-6.7 \pm 5.9 \times 10^{-10}$ & $-7.2 \pm 5.9 \times 10^{-10}$ \\
\hline$L_{1} /\left(L_{1}+L_{2}\right)_{R}$ & $0.5723 \pm 0.0023$ & $0.5727 \pm 0.0024$ & $0.6699 \pm 0.0038$ \\
\hline$L_{1} /\left(L_{1}+L_{2}\right)_{I}$ & $0.5689 \pm 0.0023$ & $0.5689 \pm 0.0024$ & $0.6700 \pm 0.0038$ \\
\hline$\ell_{3}(R$ band $)$ & 0.01385 & 0.01625 & 0.01385 \\
\hline$\ell_{3}(I$ band $)$ & 0.05125 & 0.05829 & 0.05125 \\
\hline$g_{1}$ & 0.30 & 0.30 & 0.30 \\
\hline$g_{2}$ & 0.30 & 0.30 & 0.30 \\
\hline spot 1 latitude & 1.250 & 1.250 & 1.250 \\
\hline spot 1 longitude & 0.0076 & 0.0076 & 0.0076 \\
\hline spot 1 radius & 0.278 & 0.278 & 0.278 \\
\hline spot 1 temp. factor & 0.9000 & 0.9000 & 0.9000 \\
\hline spot 2 latitude & 2.738 & 2.738 & 2.738 \\
\hline spot 2 longitude & 3.9466 & 3.9466 & 3.9466 \\
\hline spot 2 radius & 0.276 & 0.276 & 0.276 \\
\hline spot 2 temp. factor & 0.9000 & 0.9000 & 0.9000 \\
\hline
\end{tabular}

Note. Case I adopts zero light for the $D$ component, Case II adopts $D$ 's measured light (Beuzit et al. 2004), and both cases require $L_{2} /\left(L_{1}+L_{2}\right)$ to match Ribas (2003)' spectroscopic value. "Ordinary Solution" means that $L_{2} /\left(L_{1}+L_{2}\right)$ is not required to match the spectroscopic value. Quantities with standard errors were adjusted by least squares except for masses $m_{1,2}$ and radii $R_{1,2}$, which are functions of adjusted parameters. Third light, $\ell_{3}$, is in system light $\left(\ell_{1}+\ell_{2}+\ell_{3}\right)$ at phase 0.215 . Results are from simultaneous all-data solutions that pertain to $\Omega_{1}=19.530$ for Case I and $\Omega_{1}=19.810$ for Case II, because those examples lie essentially at the spectroscopic luminosity ratios. See Section 7.9 for a discussion of uncertainties in the star radii. Spot latitude runs from 0 at the $+z$ pole to $\pi$ radians at the $-z$ pole. Spot longitudes (in radians) run counter-clockwise from zero at the line of star centers as seen from above the $+z$ pole. Spot temperature factors are dimensionless, relative to the underlying unspotted surface. Gravity effect parameters, $g_{1,2}$ are exponents of bolometric flux rather than of effective temperature, as in the alternative formulation in terms of a temperature exponent $\beta$, with $g=4 \beta$.

\subsection{Weighting}

All of our solutions were simultaneous in the Ribas $R$ and $I$ light curves along with the Delfosse et al. and Table 1 RV's, so proper curve-dependent weighting of the observations is important. The assignment of weights followed precepts in Wilson (1979) that are also covered in the EB textbook by Kallrath \& Milone (2009) and in the model documentation. Curve-dependent weighting was based on the developing standard deviations of the several curves as the iterations progressed (see the discussion in Section 5.2 of Wilson \& Van Hamme 2014).

\subsection{Light from Companions}

Section 2 of Ribas (2003) gives measured $R$ and $I$ differential magnitudes between the photometrically unresolved $B C$ component (i.e., $\mathrm{CV} \mathrm{Cnc}$ ) and the photometrically unresolved $A D$ component that includes $\mathrm{CU} \mathrm{Cnc}$, then gives calculated $\ell_{3}$ fractions of total flux of the multiple system at phase $0.215 .^{8}$ The fractions are $21 \%$ in $V, 23 \%$ in $R$, and $25 \%$ in $I$. Since we

\footnotetext{
8 Here "third light," $\ell_{3}$, means the sum of all contributions to the light curves other than EB CU Cnc.
}

have $\ell_{1}+\ell_{2}+\ell_{3}$ in each band from the light curves after magnitude to flux conversion, the corresponding $\ell_{3}$ can be calculated from the Ribas observations at and near phase 0.215 for entry into the DC solution process. Ribas' differential magnitudes of the entire multiple system relative to comparison star HD 72093 were entered into DC exactly as in Ribas' Tables 1 and 2 and were converted to fluxes by the program via $F_{R}=10^{-0.4 \Delta R}$ and $F_{I}=10^{-0.4 \Delta I}$. The entered numbers for $\left(\ell_{3}\right)_{R}$ and $\left(\ell_{3}\right)_{I}$ are in Table 4.

\subsubsection{The D Component}

The other known component, $D$, turns out to have a significant impact on some parameter results through its contribution to third light and, in particular, on the relative luminosities and on the radii. Beuzit et al. (2004) measured D's magnitude relative to $A$ (i.e., to $\mathrm{CU} C \mathrm{Cnc}$ ) as $3^{\mathrm{m}} \cdot 2$ via adaptive optics, corresponding to $D$ giving about $5 \%$ as much light as $\mathrm{CU}$ Cnc. However, this seemingly minor contribution is $5 \%$ of $A$, the brightest source in the multiple system, so $D$ could add considerably to $\ell_{3}$ (percent-wise), which is much less bright than $A$ even though $\ell_{3}$ arises from several stars. Note that the fractional third light estimates adopted by Ribas came from 
photometrically measured ratios of $B C$ light to $A D$ light, since the $A D$ and $B C$ subsystems were resolved only by adaptive optics, not photometrically. Knowledge of $D$ 's existence at known brightness can allow for the removal of its light from $A D$ and the addition of that light to $B C$, as the relevant $\ell_{3}$ fraction is (symbolically) $B C D / A B C D$. Without consideration of $D$, the fraction would have been symbolized as $B C / A B C$, but the sum $B C$ would be only part of $\ell_{3}$ (lacking $D$ ), while $A$ would be too large (being unduly credited with $\ell_{D}$, a third light quantity).

\subsubsection{The D Component's Role Quantified}

The adaptive optics brightness ratio, $\ell_{D} / \ell_{A}$, was not measured in $[R, I]$ but at about 2.2 microns, corresponding to the $K$ band, so caution is needed in $\ell_{D} / \ell_{A}$ 's use within $[R, I]$ light-curve applications. Without direct knowledge of $D$ 's evolutionary state, the corresponding $R$ and $I$ ratios must be based on reasonable suppositions. A likely case assumes $D$ to be a main-sequence star of even lower mass and temperature than CU Cnc's components, since $D$ is more than $3^{\mathrm{m}}$ fainter than CU Cnc in the $K$ band. If a binary, $D$ would likely be a pair of brown dwarfs. At lower temperature than $A, D$ would be less prominent in $[R, I]$ than in the $K$ band, so $D$ 's contribution to $A D$ in $R$ and $I$ would be less than $5 \%$ and possibly much less. Could $D$ be a high-temperature source, in particular, a white dwarf? That idea is contradicted by CU Cnc's range of $U$-band magnitudes in the General Catalog of Variable Stars (Samus et al. 2010), from $10^{\mathrm{m}} \cdot 9$ to $13^{\mathrm{m}} \cdot 9$. While the top of the range might suggest a $u v$-bright hot component, the bottom rules out any steady hot and relatively bright star. Since we are dealing with known flare stars, the bright end of the $U$ range probably corresponds to one or several flares. Accordingly, two simple cases are computed here. Case $\mathrm{I}$ is that $D$ 's contribution to $\ell_{A D}$ is negligible, while Case II is that the contribution is the same as in the $K$ band. Reality probably lies in between.

To evaluate $R$ - and $I$-band ratios of total third light to total system light it is helpful to define symbols

$$
h=\frac{\ell_{\mathrm{BC}}}{\ell_{A}+\ell_{D}+\ell_{\mathrm{BC}}}
$$

and

$$
g=\frac{\ell_{D}}{\ell_{A}}
$$

as these ratios have been measured. Dimensionless ratio $h$ is conceptually the same as Ribas' $F_{3}^{R, I}$, although it is now written above to show $\ell_{D}$ explicitly. Being a directly measured quantity, $h$ simply comes from band dependent magnitude differences in the Ribas paper. Its meaning can be generalized to accomodate any future discovery of a photometrically unresolved companion to $\mathrm{CV}$ Cnc (the $B C$ components) that may be designated, say, component $E$, by addition of $\ell_{E}$ to the numerator and denominator of Equation (1). Dimensionless ratio $g$ (Beuzit et al. 2004) is calculated from the $3 .^{m} 2$ magnitude difference between $A$ and $D$ mentioned above. Its meaning can be generalized to include any future discovery of a photometrically unresolved companion (called component Q) to $A$ by addition of $\ell_{Q}$ to the numerator of Equation (2). The $h$ and $g$ ratios can be combined to give $\mathcal{R}$, the ratio of "all third light" to total system light (now including $\ell_{D}$ ) via

$$
\mathcal{R}=\frac{\frac{h}{1-h}(g+1)+g}{\frac{h}{1-h}(g+1)+g+1} .
$$

Equation (3), with input of $g=10^{3 \cdot 2 / 2.5}$ from adaptive optics, and Ribas' $h=10^{1 \mathrm{~m} \cdot 45 / 2.5}$ in $V$ band or $h=10^{1.20 / 2.5}$ in $I$ band, gives $\mathcal{R}_{V}=0.248$ and $\mathcal{R}_{I}=0.286$. Input of $g=0.000$, corresponding to vanishingly small $\ell_{D}$, gives $\mathcal{R}_{V}=0.208$ and $\mathcal{R}_{I}=0.249$, which round to Ribas' numbers. ${ }^{9}$ So accounting for $\ell_{D}$ raises estimated $\mathcal{R}_{V}$ by $15 \%$ and $\mathcal{R}_{I}$ by $19 \%$. Whether these increases significantly affect the derived $\mathrm{CU}$ Cnc masses and radii was tested and led to a definite answer, given in Section 7.8.

\subsection{Stepped Solutions}

Experiments showed the expected, rather steep, dependence of component luminosity ratio on relative star size in the stepped solutions, thus underscoring the usefulness of the luminosity ratio as an indicator of relative radii. Although the strategy for our stepped simultaneous solutions was the same as in Ribas (2003), some details differ, mainly in regard to input of the new $\mathrm{RVs},{ }^{10}$ slightly changed treatment of gravity brightening (a.k.a. darkening), somewhat different spot configurations, and adoption of $[\mathrm{M} / \mathrm{H}]=0.50$ instead of 0.00 (see Section 6.1). A minor difference from Ribas' procedure is in our use of individual light-curve points rather than Ribas' fourpoint means. The stepped quantity is $\Omega_{1}$, star 1 's surface potential parameter. A common practice with physical EB models is to adopt $\Omega_{1,2}$ or equivalent parameters as proxies for star radii, since they characterize each surface by one number rather than by a function of latitude and longitude. Although initial stages of convergence seemed excellent, a slow drift remained after 15 iterations in some solutions. Having noticed this very slow creep from a nearly converged state (corrections are small but consistently the same sign) to a fully converged state, we routinely continued for many iterations-sometimes up to 35. Our simultaneous fit to the Delfosse et al. (1999a) velocities and Ribas et al. (2003) light curves appear satisfactory, as shown in Figures 5 and 6. Light-curve standard deviations ( $\sigma$ 's) for the solutions of Table 4 were, respectively, about 0.013 and 0.008 for stars 1 and 2 in the $R$ and $I$ bands. The RV $\sigma$ 's were, respectively, 1.22 and $0.99 \mathrm{~km} \mathrm{~s}^{-1}$ for components 1 and 2 .

\subsection{CU Cnc's Derived Radii}

Although our reassignment of the $D$ component's flux contribution increases $\ell_{3}$ in the analyzed bands by almost $20 \%$, as estimated in Section 7.6.2, the effect on radii is small and is essentially nil for most other parameters, as seen in Table 4. To know this, it was necessary to follow Cases I (neglecting the $D$ star' light) and II (including the $D$ star's light in $\ell_{3}$ ) all the way to completion. Note that there are very significant parameter changes if one simply alters the input $\ell_{3}$, but the changes mainly disappear between solutions that are required to have the spectroscopically observed $R$ and $I$ luminosity ratios. Our Case I radii are smaller than those in Ribas (2003) by about

\footnotetext{
9 The corresponding $R$-band numbers are interpolated between $V$ and $I$ according to the band effective wavelengths.

${ }^{10}$ Now with direct input of the Delfosse et al. (1999b) RV's, Table 1's RV's, and Ribas (2003) R, I light curves in simultaneous solutions.
} 

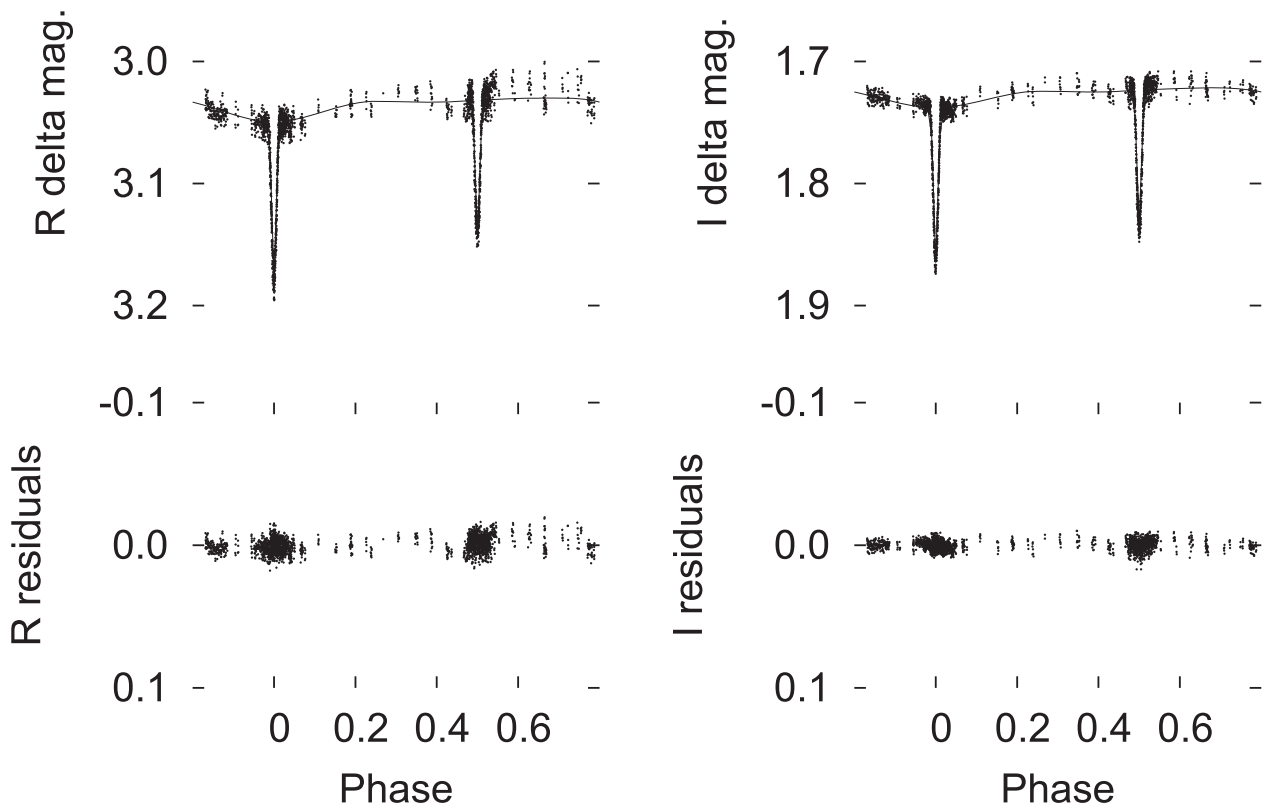

Figure 6. Case I $R$ band (left) and $I$ band (right) light curves by Ribas (2003) and the solution curves of Section 7. Case II would look the same to an eye inspection.

$2.3 \%{ }^{11}$, leaving them still above the Baraffe et al. (1998) Zero Age Main Sequence (ZAMS), as seen in Figure 7. The plotting symbols for components 1 and 2 overlap with those for the Ribas solution, thus lending visible support to the closeness of the older and newer results. For Case II (taking account of the $D$ component's contribution to $\ell_{3}$ ), $R_{1}+R_{2}$ is $3.5 \%$ smaller here than in Ribas.

\subsection{Uncertainties in CU Cnc's Derived Radii Explored}

The parameter $\sigma$ 's of Table 4 are from the simultaneous light/velocity solution that corresponds to the fourth dot from the left in each panel of Figure 4 (the dot at the spectroscopic luminosity ratio), and are computed as usual from the covariance matrix of the normal equations in the last iteration. These formal $1 \sigma$ uncertainties in $R_{1,2} / R_{\odot}$ are several times smaller than Ribas' uncertainty estimates for the radii. Because of the considerable interest in red dwarf radii, we ran test solutions to find changes in $R_{1,2} / R_{\odot}$ that ensue if $L_{2} / L_{1}$ varies over Ribas' stated uncertainty range (0.69-0.79 in $R$ band, $0.71-0.81$ in $I$ band), which is a likely basis for the radius uncertainties in Ribas' Table 3. Since the bandpass luminosity ratio is an auxiliary output quantity rather than input to our solutions, $L_{2} / L_{1}$ cannot just be changed by \pm 0.05 to ascertain corresponding radius changes, so iteration might seem necessary. However, it is much simpler to interpolate (from the stepped potential solutions that had already been done) the changes in $\Omega_{1}$ that change $L_{2} / L_{1}$ by \pm 0.05 , since $\Omega_{1}$ is an ordinary input parameter. Then, only two extra solutions, corresponding to the middle $L_{2} / L_{1} \pm 0.05$ are needed. These two solutions were very informative, showing that some parameters are virtually unaffected by luminosity ratio excursions, most others are affected only slightly, and only the radii are much changed. As expected, the sum of the radii is almost unchanged (only about $0.4 \%$ between the extremes of $L_{2} / L_{1}$ or $0.2 \%$ from the middle). The $\sigma$ 's that result from this exercise for $R_{1,2} / R_{\odot}$, of 0.0092 in $R$ band and 0.0072 in $I$ band, differ somewhat from those in Ribas' Table 4 . However, one is

\footnotetext{
$\overline{{ }^{11} \text { Comparing corresponding sums } R_{1}+R_{2}}$.
}

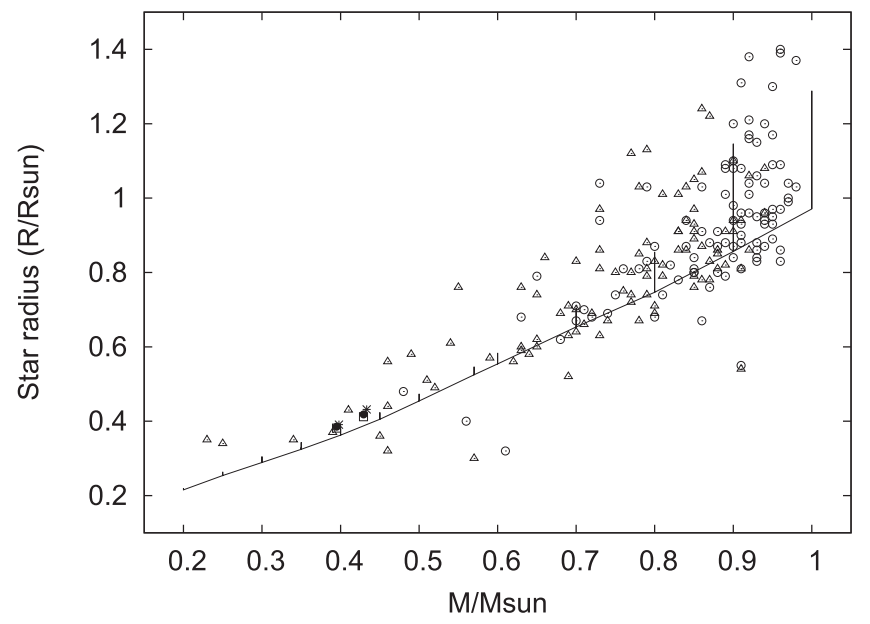

Figure 7. Radius estimates vs. mass estimates for 95 EB's with at least one $\mathrm{M}$ dwarf tabulated by Coughlin et al. (2011). Circles and triangles, respectively, represent primaries and secondaries (by mass). The two dots are for CU Cnc's components according to our Case I solution, while the two asterisks slightly above and to the right of the dots are the corresponding results from Ribas (2003) that overlap with our points. The partly obscured hollow squares just below the Case I dots mark our Case II solution (see Section 7.6). The overall $1 \sigma$ uncertainty of the $\mathrm{CU}$ Cnc points is about the radius of a dot. The sloping line is the ZAMS and the upward spikes are evolution tracks up to $12.6 \mathrm{Gyr}$, as tabulated by Baraffe et al. (1998). For masses similar to those in CU Cnc $(\approx 0.4$ $\left.M_{\odot}\right)$ and "cosmological" ages, the tracks show that evolutionary expansion is slight but appreciable compared to the observed statistical variation. However, evolutionary expansion would be negligible for ages typical of Galactic disk stars with masses similar to CU Cnc's components. See Section 8.2 for comments on CU Cnc's age.

larger than Ribas' corresponding $\sigma$ and the other is smaller and on average the difference from Ribas' uncertainties is small. $R_{1}$ and $R_{2}$, respectively, differ from Ribas' radii by $1.4 \sigma$ and $0.6 \sigma$. The "bottom line" is that the formal $\sigma$ 's of Table 4 serve well for most parameters, while Ribas' relatively large $\sigma$ 's, that mainly follow from uncertainty in the luminosity ratio, are more realistic for the radii. Having both sets of $\sigma$ 's for the radii, readers may prefer to adopt the root-mean-square values of $\sigma_{R}=0.0093$ and $\sigma_{I}=0.0074$ for star 1 and $\sigma_{R}=0.0095$ and 
$\sigma_{I}=0.0076$ for star 2 , which are just slightly larger than Ribas' estimates. In overview, the derived mean radii, $\left(R_{1}+R_{2}\right) / 2$, approximately agree with those by Ribas (2003), differing at about the $2 \sigma$ level, with the new values smaller. See Section 7.8 for differences in radii between Cases I and II.

\section{THE RED DWARF RADIUS ANOMALY—REAL OR APPARENT?}

\subsection{Overview}

Theories to account for oversized red dwarfs are given in several papers cited above, with a succinct review and references in Torres (2013). There seems to be a consensus that the anomaly is not just apparent (for example, possibly arising from measurement bias in temperature), since the anomaly persists when observational and theoretical radii are compared directly for the same masses (Torres \& Ribas 2002; Chabrier et al. 2007; Coughlin et al. 2011; Feiden \& Chaboyer 2013; Torres 2013), and also persists when two notable outliers, YY Gem and UV Psc B are disregarded. Whether these outliers might be astrophysically anomalous (perhaps unexpectedly pre or post zero age) or difficult examples for analysis is not yet clear. The anomaly also remains when the sum or average of component radii is compared to the same quantity from models (see Figure 7 of Coughlin et al. 2011 and our Section 5). However the oversize effect is only around 5\%-7\% for the two mentioned outliers and more typically around $3 \%$, while solutions are subject to difficulties connected with spots, flares, and (in some cases, shallow) partial eclipses. Chen et al. (2014) recently explored the issue of whether other structure and evolution computer models might give the slightly higher zero age radii needed to resolve the anomaly, and their Figure 2 has radius versus mass curves for four such models that have a variety of atmosphere and structural treatments. $\left[R / R_{\odot}, M / M_{\odot}\right]$ points for CU Cnc's two components from our Table 4 lie a few percent in radius above the ZAMS curves by Chen et al. (2014) and by Baraffe et al. (1998), while lying essentially on or just slightly above the relations by Spada et al. (2013) and by Dotter et al. (2008). On the observational side (Section 7.8), conversion of the $D$ component's light from its former role of being "attached" to the eclipsing system to being part of $\ell_{3}$ makes only small changes in CU Cnc's radii, but is of some interest because the changes decrease the radius anomaly, which was already small.

\subsection{Perspective on the Role of Evolutionary Expansion}

Ribas (2003) has estimated CU Cnc's age to be $320 \pm 80$ Myr from inferred membership in the Castor Moving Group (CMG). At this age, ordinary models of CU Cnc's components would be practically on the ZAMS and evolutionary expansion would be thoroughly negligible. However, Mamajek et al. (2013) argue that the CMG “...is comprised of stars from different birthsites rather than a coeval system and hence "membership" to the CMG does not provide useful age constraints". Still, the fact that CU Cnc's local space velocity is small (Feiden \& Chaboyer 2013) excludes ages at which evolutionary expansion would be significant. At an age of, say, $10^{10}$ years, evolutionary expansion could account for a significant part of CU Cnc's supposed very small radius anomaly, as seen by comparing the 12.6 billion year tracks in Figure 7 (Baraffe et al. 1998) with CU Cnc's dots. However, such an age is implausible because of the system's Population I space velocity. The figure emphasizes the importance of the few very low-mass red dwarf EB's for testing the reality of oversized radii. So does CU Cnc testify for or against reality of the radius anomaly? For now, the evidence for a real effect in this system seems marginal at best. The component radii are slightly above some versions of the ZAMS, while essentially coincident with others. Accurate data are needed on other eclipsing $\mathrm{M}$ dwarfs, preferably of essentially zero age.

\section{DIRECT DISTANCE ESTIMATION FOR CU CNC? NOT AT THIS TIME}

We examined the feasibility of carrying out a photometric/ spectroscopic distance solution with the Direct Distance Estimation algorithm (Wilson 2008; Wilson \& Van Hamme 2010; Milone \& Schiller 2013, 2015, 2017; Wilson \& Raichur 2011). That exercise is not appropriate now, due to the W-D program's present stellar atmosphere limitation to temperatures above $3500 \mathrm{~K}$, given CU Cnc's temperatures around $3100 \mathrm{~K}$, and to blackbody surfaces being much brighter than stellar atmospheres in the $R$ and $I$ bands near $3100 \mathrm{~K} .^{12}$ This problem has little effect on ordinary solutions but does strongly influence distance solutions, which require accurate modeling of surface brightness. This limitation should be removed within the next year.

\section{AUTOMATED SPEED, AUTOMATED THOROUGHNESS}

Small number statistics for low-mass dwarfs are still a problem, as shown by Figure 7, although much improved from a decade ago. Exploration of analytic pathways (i.e., light and RV curve processing) may possibly lead to re-assessing the anomaly. Thorough analysis of observed red dwarf binaries could take "forever," as the number of well-observed systems is growing much faster than traditional analysis can keep up. Fortunately, several ways to automate solutions and/or reduce processing time have appeared recently. Part of the overall solution problem, especially for red dwarfs, is to assess how well radii can realistically be determined, as exemplified by the Ribas paper. EBs with two M stars, viewed as a class, have a particular analysis problem related to the nearly equal radii, as total-annular eclipses are very unlikely. Also, most of those systems have eccentric orbits, adding several "difficult" parameters.

Contributions on automated processing of EB data sets have been appearing, some with emphasis on computational speed, others on thoroughness, avoidance of mistakes, and/or saving of personal time. There are also pipeline programs (e.g., Devor 2005) that aim to carry out the entire selection and analysis process for large data sets, including rejection of inappropriate candidates, EB-type identification, preliminary solutions, and marking of interesting examples for follow-up. An intrinsically slow scheme that runs unattended can be valuable if very thorough or accurate. Genetic algorithms (e.g., Canto et al. 2009; Coughlin et al. 2011 and contained references) are slow but look everywhere within allowed regions of parameter space for optimal solutions.

Neural networks, based on computer programmed selflearning, are ultra-fast once the learning has been completed (Prsa et al. 2008; Guinan et al. 2009; Devinney et al. 2010).

\footnotetext{
12 The program interpolates between a $3500 \mathrm{~K}$ atmosphere and a $1500 \mathrm{~K}$ blackbody in the 3500-1500 K range. (Van Homme \& Wilson 2003)
} 
The program learns by iterated fitting of synthetic light curves for which the parameter values are known. Applications so far have been limited to relatively coherent collections of systems and the intent has been to produce approximate numbers, but Prsa et al. (2008) have made thorough tests on many real EBs with impressive results. Somewhat related, in practice, to neural network solutions are those by direct comparison with archives (Wyithe \& Wilson 2001, 2002; Wilson \& Wyithe 2003), where the learning process is replaced by storage of many synthetic light curves, thus obviating the need for major number crunching, as ordinarily encountered in synthesis of EB observables. Comparison of neural network and/or archive results with those published for red dwarf EBs by Coughlin et al. (2011) via genetic algorithm would be interesting.

The genetic algorithms, neural networks, and archive-based solutions, to date, have focussed on light curves, treating RV curves as separate entities for solution. Actually, most of the utilized EBs have lacked RV curves. Many regular solutions in the literature (i.e., not genetic, neural, archive, etc.) ensure consistency via simultaneous light and velocity solutions. Kang (2010) and Kang et al. (2011) take an intermediate route by including velocities in automated large data set iterations of the W-D program.

The strategy of solutions by "Expert Systems"13 is to store the coalesced intuition of several or many expert persons within an automated binary system modeling and analysis program, as a formal algorithm. That has not yet been done but would be a platform for development into the future, based on experience gathered in its use. Part of the expertise can consist of assessments regarding what can realistically be found from the data.

Activity over the past decade suggests that exploration of all these routes to red dwarf radii will continue.

The assistance of Kristie Nault, Richard Joyce, Colette Salyk, Ken Hinkle, Karen Butler, and Christian Soto at Kitt Peak was invaluable for our Phoenix observations with the 2.1 and $4 \mathrm{~m}$ telescopes. We are grateful to the Astrophysical Research Consortium and particularly to Jack Dembicky for his assistance during our observing run on the ARC $3.5 \mathrm{~m}$ telescope. Discussions with W. Van Hamme, as well as the referee's long and thoughtful report that led to several improvements, are much appreciated. This research has made use of the NASA Astrophysics Data System Bibliographic Services, as well as the SIMBAD database, operated at CDS, Strasbourg, France. This work has made use of the VALD database, operated at Uppsala University, the Institute of Astronomy RAS in Moscow, and the University of Vienna. It is based on observations at Kitt Peak National Observatory, National Optical Astronomy Observatory (NOAO Prop. IDs: 2013B-0621 and 2014A-0147; PI: C. Pilachowski), which is operated by the Association of Universities for Research in Astronomy (AURA) under cooperative agreement with the National Science Foundation. C.A.P. acknowledges the generosity of the Kirkwood Research Fund at Indiana University. The $\mathrm{BVI}_{\mathrm{C}}$ photometry was made possible by the AAVSO Photometric All-Sky Survey (APASS), funded by the Robert Martin Ayers Sciences Fund.

Facilities: ARC: 3.5m (ARCES), KPNO: Mayall, KPNO:2.1.

\footnotetext{
${ }^{13}$ See Wilson (2005) or many books and websites for essentials.
}

\section{REFERENCES}

Baraffe, I., Chabrier, G., Allard, F., \& Hauschildt, P. H. 1998, A\&A, 337, 403 Bessell, M. S. 1979, PASP, 91, 589

Beuzit, J.-L., Segransan, D., Forveille, D., et al. 2004, A\&A, 425, 997

Cakirli, O., Ibanoglu, C., \& Dervisoglu, A. 2010, RMxAA, 46, 363

Cakirli, O., Ibanoglu, C., \& Gungor, C. 2009, NewA, 14, 496

Cakirli, O., Ibanoglu, C., \& Sipahi, E. 2013, MNRAS, 429, 85

Canto, J., Curiel, S., \& Martinez-Gomez, E. 2009, A\&A, 501, 1259

Chabrier, G., Gallardo, J., \& Baraffe, I. 2007, A\&A, 472, L17

Chen, Y., Girardi, L., Bressan, A., et al. 2014, MNRAS, 444, 2525

Coughlin, J. L., Lopez-Morales, M., Harrison, T. E., Ule, N., \& Hoffman, D. I. 2011, AJ, 141, 78

Cram, L. E., \& Mullan, D. J. 1985, ApJ, 294, 626

Delfosse, X., Forville, T., Beuzit, J.-L., et al. 1999a, A\&A, 344, 897

Delfosse, X., Forville, T., Mayor, M., Burnet, M., \& Perrier, C. 1999b, A\&A, 341, L63

Devinney, E. J., Prsa, A., \& DeGeorge, M. 2010, ASPC, 282, 57

Devor, J. 2005, ApJ, 628, 411

Dimitrov, D. P., \& Kjurkchieva, D. P. 2010, MNRAS, 406, 2559

Dotter, A., Chaboyer, B., Jevremovic, D., et al. 2008, ApJS, 178, 89

Feiden, G. A., \& Chaboyer, B. 2013, ApJ, 779, 183

Gomez, Y., Morales, J. C., Faedi, F., et al. 2014, A\&A, 572, 50

Guinan, E. F., Prsa, A., Devinney, E. J., \& Engle, S. G. 2009, ASPC, 404, 361

Gustafsson, B., Edvardsson, B., Eriksson, K., et al. 2008, A\&A, 486, 951

Haro, G., Chavira, E., \& Gonzalez, G. 1975, IBVS, 1031

Helminiak, K. G., Konacki, M., Złoczewski, K., et al. 2011, A\&A, 527, 14

Henden, A. A., Levine, S. E., Terrell, D., Smith, T. C., \& Welch, D. 2012, JAVSO, 40, 430

Irwin, J., Charbonneau, D., Berta, Z. K., et al. 2009, ApJ, 701, 1436

Jankovics, I., Tsvetkova, K. P., \& Tsvetkov, M. K. 1978, IBVS, 1454

Janson, M., Bergfors, C., Brandner, W., et al. 2014, ApJ, 789, 102

Jester, S., Schneider, D. P., Richards, G. T., et al. 2005, AJ, 130, 873

Kallrath, J., \& Milone, E. F. 2009, Eclipsing Binary Stars: Modeling and Analysis (New York: Springer)

Kang, Y. 2010, JASS, 27, 75

Kang, Y., Hong, K. S., \& Rittipruk, P. 2011, in Proc. IAU 282, Ninth Pacific Rim Conference on Stellar Astrophysics, ed. S. B. Qian et al. (San Francisco, CA: ASP), 329

Macdonald, J., \& Mullan, D. J. 2014, ApJ, 787, 70

Mamajek, E. E., Bartlett, J. L., Seifahrt, A., et al. 2013, AJ, 146, 154

Milone, E. F., \& Schiller, S. J. 2013, in IAU Symp. 289, Advancing the Physics of Cosmic Distances, ed. R. de Grijs (Cambridge: Cambridge Univ. Press), 227

Milone, E. F., \& Schiller, S. J. 2015, IAUGA, 22, 2243715

Milone, E. F., Schiller, S. J., \& Amby, T. O. 2017, AJ, submitted

Morales, J. C., Gallardo, J., Ribas, I., et al. 2010, ApJ, 718, 502

Nefs, S. V., Birkby, J. L., Snellen, I. A. G., et al. 2013, MNRAS, 431, 3240 Petersen, B. R. 1982, IBVS, 2141

Prsa, A., Guinan, E. F., Devinney, E. J., et al. 2008, ApJ, 687, 542

Qian, S.-B., Zhang, J., Zhu, L. Y., et al. 2012, MNRAS, 423, 3646

Ribas, I. 2003, A\&A, 398, 239

Rozyczka, M., Kaluzny, J., Pietrukowicz, P., et al. 2009, AcA, 59, 385

Ryabchikova, T., Piskunov, N., Kurucz, R. L., et al. 2015, PhyS, 90, 054005

Samus, N. N., Kazarovets, E. V., Kireeva, N. N., Pastukhova, E. N., \& Durlevich, O. V. 2010, General Catalog of Variable Stars (Moscow: Odessa Astronomical Publications)

Sneden, C. 1973, ApJ, 184, 839

Spada, F., Demarque, P., Kim, Y. C., \& Sills, A. 2013, ApJ, 776, 87

Stauffer, J. R., Balachandran, S. C., Krishamurthi, A., et al. 1997, ApJ, 475,604

Tonry, J., \& Davis, M. 1979, AJ, 84, 1511

Torres, G. 2013, AN, 334, 4

Torres, G., \& Ribas, I. 2002, ApJ, 567, 1140

Van Hamme, W., \& Wilson, R. E. 2003, in ASP Conf. Ser. 298, GAIA Spectroscopy: Sciece and Technology, ed. U. Manari (San Francisco, CA: ASP), 323

Wallace, L., Hinkle, K., \& Livingston, W. 1993, National Solar Observatory Technical Rep. 93-001

Wallace, L., \& Livingston, W. 1992, N.S.O. Technical Rep. No. 92-001, (National Solar Observatory: Tucson)

Wilson, R. E. 1979, ApJ, 234, 1024

Wilson, R. E. 1990, ApJ, 356, 613

Wilson, R. E. 2005, Ap\&SS, 296, 197

Wilson, R. E. 2007, ASPC, 362, 3

Wilson, R. E. 2008, ApJ, 672, 575 
Wilson, R. E. 2012a, JASS, 29, 115

Wilson, R. E. 2012b, AJ, 144, 73

Wilson, R. E., \& Devinney, E. J. 1971, ApJ, 166, 605

Wilson, R. E., \& Raichur, H. 2011, MNRAS, 415, 596

Wilson, R. E., \& Van Hamme, W. 2009, ApJ, 699, 118

Wilson, R. E., \& Van Hamme, W. 2010, ASPC, 435, 45

Wilson, R. E., \& Van Hamme, W. 2014, ApJ, 780, 151
Wilson, R. E., Van Hamme, W., \& Terrell, D. 2010, ApJ, 723, 1469

Wilson, R. E., \& Wyithe, J. S. B. 2003, ASPC, 298, 313

Windmiller, G., Orosz, J. A., \& Etzel, P. B. 2010, ApJ, 712, 1003

Wyithe, J. S. B., \& Wilson, R. E. 2001, ApJ, 559, 260

Wyithe, J. S. B., \& Wilson, R. E. 2002, ApJ, 571, 293

Zhang, L., Pi, Q., \& Yang, Y. 2014, MNRAS, 442, 2620

Zhou, G., Bayliss, D., Hartman, J. D., et al. 2014, MNRAS, 437, 2831 